\title{
Maternal Prenatal Microbiome and Infant's Immune System at the Origins of the Development of Health and Disease
}

\section{Microbioma Materno Prenatal y Sistema Inmune del Lactante en los Orígenes del Desarrollo de la Salud y Enfermedad}

XVIII International Seminar on Health, Food and Human Nutrition

Corresponding Author:

Leslie Gricel Cuzco Macías

lesliecuzco@gmail.com

Published: 9 September 2021

Production and Hosting by

Knowledge $E$

(c) Iván Enrique Naranjo Logroño et al. This article is distributed under the terms of the Creative Commons Attribution License, which permits unrestricted use and redistribution provided that the original author and source are credited.

\author{
Iván Enrique Naranjo Logroño, ${ }^{1,2}$, Leslie Gricel Cuzco Macías ${ }^{1,3}$, Alison Tamara \\ Ruiz Chico ${ }^{1}$, and Anthony Alfonso Naranjo Coronel ${ }^{2}$ \\ 1Departamento de Ginecología, Carrera de Medicina, Facultad de Salud Pública, Escuela \\ Superior Politécnica de Chimborazo, Riobamba, Ecuador \\ ${ }^{2}$ COLPOMED Centro, Hospital del día, Riobamba, Chimborazo, Ecuador \\ ${ }^{3}$ Hospital Provincial General Docente de Riobamba, Riobamba, Ecuador
}

\section{Abstract}

Introduction: The human microbiome refers to the presence of microorganisms that live with its host. Objective: To analyze the relationship between the maternal perinatal microbiome and the development of the infant's immune system, at the origins of the development of health and disease. Methodology: A non-systematic bibliographic review was carried out, including those controlled and randomized clinical trials focused on the relationship of the prenatal maternal microbiome and the infant's immune system. And all those works whose approach was different from the topic raised were excluded. Discussion: 20 min after birth, the microbiome of newborns by vaginal delivery resembles the microbiota of their mother's vagina, while those born by caesarean section house microbial communities that are usually found in human skin. The acquisition of the microbiome continues during the first years of life, with a microbiome of the baby's gastrointestinal tract beginning to resemble that of an adult from the first year of life. Conclusion: Bacteria are microorganisms that have managed to colonize the vast majority of land surfaces, showing great adaptability. The human being is not indifferent, and hypotheses have been raised that affirm his participation in the development of health and the onset of the disease.

Keywords: microbiota, inmune system, infant nutritional physiological phenomena.

\section{Resumen}

Introducción: El microbioma humano se refiere a la presencia de microorganismos que conviven con su hospedero. Objetivo: Analizar la relación existente entre el microbioma materno perinatal y el desarrollo del sistema inmune del lactante, en los orígenes del desarrollo de la salud y enfermedad. Metodología: Se realizó una revisión bibliográfica no sistemática, donde se incluyeron aquellos ensayos clínicos controlados y randomizados enfocados en la relación del microbioma materno prenatal y el sistema inmune del lactante. Y se excluyeron todos aquellos trabajos cuyo enfoque fue diferente al tema planteado. Resultados: Se encontraron 61 fuentes bibliográficas, de las cuales se incluyeron 53 artículos que contenían la información relacionada al tema y publicados en los últimos 11 años. Discusión: 20 min después del nacimiento, el microbioma de los recién nacidos por parto vaginal se asemeja 
a la microbiota de la vagina de su madre, mientras que los nacidos por cesárea albergan comunidades microbianas que generalmente se encuentran en la piel humana. La adquisición del microbioma continúa durante los primeros años de vida, con un el microbioma del tracto gastrointestinal del bebé comienza a parecerse al de un adulto desde el primer año de vida. Conclusiones: Las bacterias, son microorganismos que han logrado colonizar la gran mayoría de las superficies terrestres, mostrando una gran capacidad de adaptación. El ser humano, no es indiferente, y se han planteado hipótesis que aseveran su participación en el desarrollo de la salud e inicio de la enfermedad.

Palabras Clave: microbiota, sistema inmunológico, fenómenos fisiológicos nutricionales del lactante.

\section{Introducción}

El microbioma humano se refiere a la presencia de microorganismos que conviven con su hospedero, en este caso el humano, ocupando sitios específicos como las superficies mucosas y de piel a lo largo del cuerpo. En una perfecta relación simbiótica, se estima que alrededor de 1014 células microbianas (10 veces más que las células humanas propias del organismo) colonizan dichas superficies [1]. Se ha demostrado que cualquier alteración tanto en la funcionalidad o composición de este pequeño ecosistema humano se asocian con el desarrollo de ciertas enfermedades humanas que afectaran al lactante en el futuro.

Los análisis de la composición del microbioma en humanos adultos han revelado numerosas asociaciones entre los filos bacterianos específicos y la enfermedad, y han dilucidado varias vías a través de las cuales esto puede ocurrir. Quizás el primer ejemplo sea la relación descrita entre el microbioma intestinal y los cambios en el nivel de los filos asociados con las vías metabólicas y la obesidad [2, 3].

Últimamente, se estudia la posibilidad que muchos de los cambios microbianos asociados a las enfermedades y sus consecuencias inmunes podrían originarse durante los primeros días de vida. Durante el periodo posnatal inmediato, el microbiota está en proceso de colonización del huésped y su composición es altamente inestable. Este tiempo de colonización temprana también se correlaciona con el desarrollo del sistema inmune y su educación, lo que le permite tolerar su ambiente para combatir a los patógenos y evitar la alergia y la autoinmunidad [4].

Hoy en día existe cada vez más evidencia científica que demuestra que ciertas poblaciones celulares especialmente las del sistema inmune pueden estar reguladas por la microbiota en un momento específico de la vida. Además, las alteraciones de la microbiota independientemente del motivo durante un periodo específico de la vida pueden tener efectos importantes sobre el sistema inmune posteriormente en la vida del lactante. 


\section{Métodos}

A través de una revisión bibliográfica no sistemática utilizando los buscadores: LILACS, CINAHL, COCHRANE, EBSCO, MEDLINE, SCIELO, SCOPUS y PUBMED, en el cual se abarcó artículos en español e inglés, con publicaciones desde el año 2008 al 2019.

Entre los términos de búsqueda se encuentran: Microbiota, sistema inmunológico, fenómenos fisiológicos nutricionales del lactante. Se incluyeron aquellos ensayos clínicos controlados y randomizados referentes a la relación existente entre el microbioma materno prenatal y el sistema inmune del lactante. $Y$ se excluyeron todos aquellos trabajos cuyo enfoque fue diferente al tema planteado.

Riesgo de sesgo poco claro.

\section{Resultados}

De la búsqueda antes descrita se encontró 61 fuentes bibliográficas acerca del microbioma materno prenatal y el sistema inmunológico del lactante, en los orígenes del desarrollo de la salud y la enfermedad, de las cuales se utilizaron 53 publicaciones científicas relevantes, los cuales aportaron hechos a favor de la investigación realizada; los artículos científicos excluidos no cumplieron con los criterios de inclusión planteados.

\section{Discusión}

El microbioma humano consiste en 10 a 100 billones de células microbianas simbióticas albergadas por cada persona, principalmente bacterias en el intestino; el microbioma humano, sin embargo, consiste en los genes que albergan estas células [5].

Se han lanzado proyectos de microbioma en todo el mundo con el objetivo de comprender los roles que desempeñan estos simbiontes y su impacto en la salud humana $[6,7]$.

Así como la pregunta ‘Qué es ser humano?' ha preocupado al hombre, desde el comienzo de la historia registrada, la pregunta ‘Qué es el microbioma humano?' o ‘Cómo se desarrolla la microbiota en el cuerpo humano?', ha inquietado a los investigadores desde que Joshua Lederberg acuñó el término en 2001 [8].

El tracto gastrointestinal de un infante humano proporciona un entorno completamente nuevo para la colonización microbiana [9]. De hecho, el microbioma que un infante comienza a adquirir depende en gran medida del modo de parto [10].

Veinte minutos después del nacimiento, el microbioma de los recién nacidos por parto vaginal se asemeja a la microbiota de la vagina de su madre, mientras que los nacidos por cesárea albergan comunidades microbianas que generalmente se encuentran en la piel humana [11]. La adquisición del microbioma continúa durante los primeros años de vida, con un el microbioma del tracto gastrointestinal del bebé comienza a parecerse al de un adulto desde el primer año de vida [12]. 
En un estudio de caso en el que se monitorizó la microbiota de un bebé durante los primeros 2,5 años de vida, la diversidad filogenética aumentó de manera significativa y lineal con el tiempo. Además, se observaron cambios significativos en la composición de la microbiota intestinal en cinco puntos de tiempo: Introducción de una dieta de la leche materna, desarrollo de fiebre en el día 92, introducción de cereal de arroz en el día 134, introducción de alimentos de fórmula y de mesa en el día 161, e introducción de tratamiento con antibióticos y dieta para adultos en el día 371 [13].

Curiosamente, cada cambio en la dieta estuvo acompañado por cambios del microbioma intestinal y el enriquecimiento de los genes correspondientes. Por ejemplo, cuando se introdujo la dieta del adulto en el bebé, los genes en el microbioma asociados con la biosíntesis de vitaminas y la digestión con polisacáridos se enriquecieron [13]. La interacción entre el microbioma humano y el medio ambiente es dinámica, y los microbios humanos fluyen libremente sobre las superficies tocadas todos los días. Fierer et al. demostraron que las yemas de los dedos humanos pueden transferir comunidades de microbios a los teclados, y que estas comunidades diferencian fuertemente a las personas [14].

Las gráficas de PCoA mostraron que era posible determinar qué dedos estaban escribiendo en qué teclas y qué personas estaban usando qué teclados: Incluso era posible vincular la mano de una persona al ratón de la computadora que usan con una precisión de hasta el 95\% en comparación con una base de datos de otras manos. En general, este estudio mostró que las comunidades microbianas se transfieren constantemente entre las superficies y que existe una interacción dinámica entre el microbioma ambiental y los diferentes sitios del cuerpo humano [15].

\subsection{Exposición prenatal}

El desarrollo del microbioma comienza mucho antes de que nazca el bebé. Contrariamente a lo que se pensaba, el líquido amniótico no es estéril $[16,17]$. En algunos casos, la presencia de bacterias en el líquido amniótico se asocia con un estado de enfermedad. Mycoplasma y Ureaplasma en el líquido amniótico son aislamientos frecuentes asociados con efectos perjudiciales para la salud, como corioamnionitis, parto prematuro y enterocolitis necrotizante (ECN) [18-20].

Además, las mujeres con infecciones vaginales son mucho más propensas a tener bebés prematuros [21]. Aparte de esto, las bacterias también se detectan a menudo en el líquido amniótico y placentas de los bebés sanos a término [22-24]. Otros filos detectados en el líquido amniótico y la placenta se superponen con los filos que se encuentran comúnmente en el microbioma oral: Firmicutes, Bacteroidetes, Actinobacteria, Proteobacteria y Fusobacteria [17, 25]

El meconio tampoco es estéril [26-28], lo que apoya la idea de que los microbios en el líquido amniótico tienen acceso al feto por nacer. Un estudio reciente comparó la microbiota de meconio en bebés prematuros con conjuntos de datos separados de microbiotas amnióticas, vaginales y de cavidad oral, y descubrió que la mayoría de las superposiciones entre el meconio era de los conjuntos de datos amnióticos [28]. Los taxones bacterianos que se encuentran en el meconio que utilizan enfoques 
independientes y dependientes del cultivo se superponen con la microbiota intestinal adulta [29].

Se detectaron enterobacterias (incluyendo Escherichia coli y Shigella spp.), Enterococos, estreptococos, estafilococos (incluyendo Staphylococcus epidermidis) y bifidobacterias en bebés sanos, de término completo [28, 30-32]. Además, la administración de Enterococcus faecium a ratas preñadas permitió el aislamiento de la misma bacteria del meconio de crías a término inmediatamente después del nacimiento por cesárea [30]. Por lo tanto, si bien la exposición a microbios vaginales patógenos puede considerarse eventos infecciosos, la exposición prenatal a microbios fecales es probablemente una parte natural del desarrollo en el útero. La forma en que estos microbios obtienen acceso al útero sigue siendo desconocida, aunque la translocación bacteriana del intestino al torrente sanguíneo y luego al útero es una teoría que se ha propuesto, pero que aún no se ha probado experimentalmente [32].

\subsection{Parto vaginal: El primer paso en la colonización microbiana postnatal}

Como sabemos, la primera exposición microbiana importante para un bebé nacido por vía vaginal es en el canal del parto, un evento potencialmente importante para establecer un microbioma saludable en una etapa temprana de la vida. La cesárea evita esta exposición y altera el grupo inicial de microbios a los que está expuesto el neonato [11].

En un estudio realizado en el 2016 se investigó el impacto del modo de administración en la maduración y diversidad del microbioma durante los primeros 2 años de vida. En comparación con los bebés nacidos por vía vaginal, los niños con cesárea mostraron una diversidad filogenética, riqueza y uniformidad significativamente mayor $(p<0,05)$ al inicio. Sin embargo, estos disminuyeron significativamente en los bebés nacidos por cesárea durante el primer mes después del nacimiento, y los niños nacidos por cesárea posteriormente mostraron menor diversidad y riqueza hasta los 2 años, especialmente después de los 8 meses de edad. Estos efectos no se debieron a diferencias en la abundancia bacteriana absoluta [33].

También esperamos que la diversidad comparativa entre comunidades (diversidad B) se altere en los recién nacidos por cesárea, lo que refleja las diferentes exposiciones microbianas durante el parto y la diversidad alterada que se observó. Para la primera evacuación intestinal de los bebés (media \pm desviación estándar, 20,2 \pm 18,3 hr de vida), la diversidad fecal no fue significativamente diferente, lo que sugiere que el microbioma en los lactantes colonizadores fue de complejidad similar. A partir de entonces, la cesárea alteró significativamente la diversidad microbiana en comparación con el parto vaginal (MANOVA permutativo de UniFrac no ponderado, $p<0,001$ ), pero representó una pequeña fracción de la variación total entre las muestras $(R=0,02)$. Para varios taxones bacterianos comunes, las abundancias se alteraron en los recién nacidos por cesárea en comparación con los nacidos por vía vaginal, lo que subyace a las diferencias en la diversidad [34]. 
De manera más prominente, la abundancia de Bacteroides fue significativamente menor en los recién nacidos por cesárea, independientemente del modo de alimentación predominante. A los 12 meses, el balance de Bacteroides, Bifidobacterium $\mathrm{k}$, Enterobacteriaceae que dominaron el primer año de vida en todos los lactantes se reemplazó por una mezcla de Firmicutes, principalmente Clostridiales [33].

Aunque las Clostridiales y las Enterobacterias en particular fueron significativamente más abundantes [tamaño del efecto del análisis discriminante lineal (LEfSe), $p<0,05$ ] en los bebés nacidos por cesárea durante el primer año, llenando el vacío dejado por Bacteroidales, pocos taxones fueron significativamente diferentes durante el segundo año de vida [34]. Esta similitud cada vez mayor después de 1 año en niños nacidos por vía vaginal o por cesárea indica que ambas comunidades experimentan una maduración gradual, pareciéndose finalmente al microbioma fecal adulta. Los taxones que dominaron en los primeros meses de vida ya sean perturbados o no por cesárea o antibióticos, disminuyeron a medida que los taxones de edad avanzada los reemplazaron [33].

La hipótesis que se planteó en el siguiente artículo Los antibióticos, el modo de nacimiento y la dieta dan forma a la maduración del microbioma durante la vida temprana, es que la interrupción de la cesárea también podría alterar los patrones de maduración del microbioma en los bebés, similar a la exposición a antibióticos [33].

Usando el modelo de maduración del microbioma descrito en los Materiales suplementarios, encontramos que los bebés con cesárea y parto vaginal mostraron grados similares de maduración del microbioma durante los primeros 6 meses de vida. Posteriormente, la maduración del microbioma se estancó en los recién nacidos por cesárea, con una caída relativa de la maduración en comparación con los bebés nacidos por vía vaginal durante el resto del período de estudio [33].

\subsection{La lactancia materna fomenta la maduración del microbioma en los primeros años de vida}

En el neonato el tracto gastrointestinal es estéril sin gérmenes conforme avanzan los días el periodo posnatal es importante para el desarrollo del microbioma ya que esta ayuda en el desarrollo del sistema inmunológico y estado de salud del ser humano, existen muchos factores que pueden alternar el correcto desarrollo del microbioma como por ejemplo si es parto normal o cesárea, lactancia materna y artificial y el uso de antibióticos. Hace algunos años se promocionaba la lactancia materna por ser estéril libre de gérmenes, pero en la actualidad está en controversia [33].

La lactancia materna en el recién nacido reduce la morbilidad y mortalidad producida por enfermedades infecciosas, debido en gran parte a sus componentes inmunológicos que protegen al infante contra infecciones. [34] La leche humana contiene su propio microbioma caracterizada por la predominancia de Proteobacterias y Firmicutes, prebióticos (por ej., oligosacáridos de la leche humana) y factores antimicrobianos, Conviene tener en cuenta que entre las bacterias aisladas normalmente de la leche materna existen algunas especies, como L. gasseri, L. plantarum, L. rhamnosus, L. salivarius, $L$. fermentum o E. faecium, que se incluyen habitualmente entre las potencialmente 
probióticas. De hecho, los estudios recientes han revelado que los lactobacilos aislados de leche materna poseen un potencial probiótico similar o superior al de ciertas cepas de lactobacilos de gran difusión comercial, como L. rhamnosus GG, L. casei immunitass o L. johnsonii. Las bacterias lácticas desempeñan un papel muy importante en las barreras microbiológicas primarias que se forman en las mucosas con el fin de prevenir infecciones. En general, las bacterias lácticas aisladas de leche materna parecen mostrar un gran potencial para adherirse a las mucosas y/o para producir sustancias antimicrobianas; se han propuesto algunas cepas como agentes bioterapéuticos para la prevención de infecciones neonatales y mastitis causadas por Staphylococcus aureus. Además, otras bacterias de la leche, como estreptococos, estafilococos y E. coli, pueden resultar muy útiles para reducir la incidencia de patógenos en neonatos de alto riesgo expuestos a ambientes hospitalarios. Por ejemplo, algunos estreptococos del grupo Viridans evitan que cepas de aureus resistentes a la meticilina puedan colonizar la cavidad oral de los niños. Por otra parte, la presencia de Streptococcus parasanguis en la cavidad oral infantil previene el desarrollo de caries y enfermedades periodontales, debido a su antagonismo con las bacterias responsables de tales procesos, como S. mutans. Por lo que respecta a E. coli, esta especie comprende cepas patógenas y también comensales; estas últimas son habitantes normales, y con funciones ecológicas relevantes, en las mucosas humanas. De hecho, la cepa $E$. coli es la base de un producto probiótico infantil ampliamente difundido en Alemania y otros países del este de Europa, y diversos estudios han demostrado que su aplicación por vía oral, tanto en niños a término como en prematuros, reduce significativamente el número y la gravedad de las infecciones infantiles, se plantea que el lactante que se alimenta con alrededor de 800 $\mathrm{mL}$ de leche al día, se expone a aproximadamente entre 105 y 107 bacterias [35].

La lactancia materna se encarga de las bases del microbioma intestinal de los lactantes por medio del contacto con los microorganismos de la areola y la leche materna, brindando fuentes de energía claves para muchas bacterias (oligosacáridos de la leche humana).

A más de ello, es esencial reconocer la diferencia composicional de este microbioma entre los lactantes amamantados y los alimentados con fórmula, con los primeros siendo poblados con mayores proporciones de Bifidobacteria y Lactobacillus spp., y los segundos poblados con una mayor prevalencia de bacterias clostridiales y proteobacterias [36].

Es así que, los niños que reciben fórmula muestran una menor diversidad bacteriana incluso después (12-24 meses de edad), consecuentemente, provocando alteraciones en el crecimiento ulterior [36].

La evidencia epidemiológica proporciona más apoyo para el beneficioso rol de la lactancia materna en la promoción de la salud infantil. La alimentación con fórmula ha sido asociada con un aumento del riesgo de varias enfermedades hiperinflamatorias y mediadas por inmunidad [36]. 


\subsection{La dieta materna asociada a la gestación da forma al micro- bioma infantil en desarrollo}

Actualmente se han realizado estudios los cuales demuestran que la formación del microbioma intestinal del recién nacido se programa desde la vida intraútero. La teoría de que durante la etapa fetal el intestino se encuentra estéril y que las exposiciones a los microorganismos maternos ocurren tras el nacimiento, ha sido modificada a la luz de noveles investigaciones, que demuestran que los primeros contactos con el microbioma intestinal materna los tiene el feto a través de la placenta y el líquido amniótico [37].

Estudios recientes han sugerido que la placenta alberga una comunidad de microbios de baja abundancia [38].

Posteriormente durante el nacimiento continúa la colonización del intestino debido al contacto de la región perianal con bacterias maternas (transferencia materno-fetal).

La información actual empleada ha servido para apoyar el rol importante de la dieta materna asociada a la gestación en el moldeo del microbioma infantil. En una cohorte prospectiva de 26 díadas madre-hijo, un alto contenido de grasa en la dieta materna gestacional se asoció con distintas variaciones en la composición microbiana del intestino neonatal (meconio), que persistieron hasta las 4 a 6 semanas de edad. Tomando en consideración, la capacidad de modular la respuesta inmunológica del microbioma en las primeras etapas de vida, y siendo influenciada principalmente por la dieta materna durante el embarazo, se hallaron frecuencias más altas de células tímicas $\mathrm{T}$ reguladoras en estos cachorros. Finalmente, demostrando el efecto de la dieta materna en la capacidad funcional del microbioma infantil, los lechones nacidos de cerdas que fueron alimentadas con una dieta occidental (dieta de alta energía, alto contenido de grasa, a base de fructosa) durante el embarazo mostraron una disminución de la producción de AGCC. En estos estudios, se apoya la importancia del microbioma como un mediador que enlaza la dieta materna durante la gestación con la salud infantil, fundamentando el papel del microbioma en los ODSE. Futuros estudios pueden arrojar más luz sobre los efectos prolongados de la dieta materna gestacional en la salud y el desarrollo [39].

\subsection{Los antibióticos alteran la colonización infantil y disminuyen la maduración del microbioma}

Los antibióticos son los medicamentos más comunes recetados para los niños. Datos epidemiológicos recientes sugieren una asociación entre el uso temprano de antibióticos y los fenotipos de enfermedades en la edad adulta. El uso de antibióticos durante la infancia induce desequilibrios en el microbioma intestinal, llamada disbiosis. Las respuestas del microbioma intestinal a los antibióticos y su posible vínculo con el desarrollo de la enfermedad son especialmente complejas de estudiar en el intestino infantil cambiante. Aquí, sintetizamos los conocimientos actuales que relacionan los antibióticos, la disbiosis y la enfermedad, y proponemos un marco para estudiar la disbiosis relacionada con los antibióticos en niños [39]. 
El intestino infantil se coloniza inicialmente con Proteobacteria y Firmicutes, seguido de un aumento gradual de Actinobacteria (potencialmente debido a la introducción de la leche materna). A los 6 meses de edad, los Bacteroidetes dominan, mientras que las Proteobacterias y Actinobacterias disminuyen gradualmente, lo que puede atribuirse a la abundancia de carbohidratos en los alimentos sólidos que coinciden con el destete [39].

El microbioma de la leche humana también cambia con el tiempo y depende del peso de la madre. Por ejemplo, Weissella, Leuconostoc, Staphylococcus, Streptococcus y Lactococcus predominan en la leche inmediatamente después de dar a luz, y la leche de madres obesas es menos diversa que la de madres no obesas. Estas bacterias ingeridas proporcionan una fuente constante de miembros de la comunidad para ayudar a colonizar el tracto gastrointestinal. Los prebióticos transmitidos por la leche que modulan las bacterias presentes en el tracto GI incluyen los oligosacáridos de la leche humana [39].

El uso excesivo de antibióticos de amplio espectro para condiciones que responden a agentes de espectro estrecho ha aumentado dramáticamente. Incluso después de ajustar las diferencias en la edad del paciente, las comorbilidades y los factores sociodemográficos, los niños con las mismas infecciones pueden recibir índices de prescripciones de antibióticos muy diferentes dependiendo de la práctica o el médico visitado [39].

El uso indiscriminado de antibióticos en pediatría parece ser ubicuo en diversos países. Tradicionalmente su uso excesivo ha sido asociado a una promoción de la resistencia bacteriana. Adicionalmente hoy se describe una potente asociación entre el uso de antibióticos en la infancia temprana y las enfermedades crónicas como el asma, la diabetes y la obesidad [40]. La administración de antibióticos durante el primer año de vida puede tener un profundo rol en el desarrollo del microbioma a través de 4 grandes mecanismos de disbiosis. La pérdida de taxas claves, la pérdida de diversidad general, los cambios en las capacidades metabólicas del microbioma y el sobrecrecimiento de patógenos se constituyen como posibles consecuencias de las disbiosis causadas por antibióticos [41].

\subsection{Las enfermedades no transmisibles asociadas a disbiosis pro- porcionan un apoyo adicional para el microbioma. Enterocoli- tis Necrotizante}

La enterocolitis necrotizante resulta ser la afección quirúrgica con mayor mortalidad presente en el período neonatal, mediante varios estudios realizados, su causa se ha centrado con frecuencia y específicamente en la alteración severa del tracto gastrointestinal. Esta afección y su desarrollo se han visto controvertidos, generalmente se le atribuye una variabilidad según los orígenes étnicos y geográficos o inclusive a factores genéticos y/o ambientales. Sin embargo, su frecuencia se ha visto mínima en países como Japón, Suiza y Austria, y mayormente frecuente en América del Norte, el Reino Unido e Irlanda [41]. 
A pesar de los grandes avances en la atención neonatal, la cifra de la tasa de mortalidad por efecto de la enterocolitis sigue siendo muy alta, estadísticamente se centra en un censo 30 a $50 \%$ de neonatos. Debido a los problemas provocados por esta afección es que justamente se necesitan con urgencia estrategias terapéuticas para preservar y/o reconstituir la estructura intestinal de los neonatos afectados [26].

Entre los años 2006 y 2007, en el Reino Unido se realizó un estudio que involucró a 158 unidades de cuidados intensivos neonatales de nivel 2 y 3 . Un total de 211 bebés fueron diagnosticados de enterocolitis necrotizante durante un período de prevalencia de $2 \%$ en ingresos en unidades de cuidados intensivos. En las últimas décadas se ha visto la gran incidencia y aumento de la enterocolitis necrotizante en Canadá y el Reino Unido, un factor que seguirá en aumento en relación con el porcentaje de bebés prematuros y de bajo peso al nacer tratados en unidades de cuidados intensivos neonatales. En la actualidad aún no existe un consenso o evidencia sobre qué régimen de antibióticos se debe prescribir para los recién nacidos con enterocolitis necrotizante. Generalmente el tratamiento no quirúrgico incluye alimentación de retención, soporte ventilatorio, reanimación con líquidos, soporte inotrópico, corrección del desequilibrio ácido-base, reposo intestinal y antibióticos, para realizar el diagnóstico de este último es necesario seguir un riguroso protocolo institucional y resultados de sensibilidad en el paciente. Para corroborar esta información se cita además el estudio retrospectivo realizado en el Hospital Pediátrico Docente 'Centro Habana' donde hubieron 63 paciente fallecidos por enterocolitis necrotizante; se encontró que $71,4 \%$ de los afectados eran de la raza blanca y el $68,2 \%$ del sexo masculino. La edad más frecuente se halló en los menores de 3 meses de edad (36,5\%) y el $46 \%$ del total de la muestra estudiada tuvo un peso al nacer inferior a los $1500 \mathrm{~g}$. La prematuridad apareció asociada en el 55,5\% de los fallecidos y el 65\% tuvo lactancia mixta desde el momento de nacimiento [42].

En cuanto las formas de prevención estudiadas, los probióticos resultan ser los organismos con mayor eficacia para prevenir enterocolitis necrotizante en lactantes con bajo peso al nacer, esto se puede confirmar mediante el estudio realizado por Cochrane; se analizaron 24 ensayos en los cuales se demostró que la suplementación con probióticos enterales redujo significativamente la incidencia de enterocolitis necrotizante grave y la mortalidad, de igual forma con un paciente crítico ingresado en terapia intensiva, la lactancia materna exclusiva resulta un tratamiento eficaz para evitar la ECN gracias a que le confiere protección inmunológica al neonato, madurez del enterocito, así como la presencia de inmunomoduladores que regulan la respuesta inmunológica a nivel intestinal $[43,44]$.

\subsection{Asma y enfermedad atópica}

Existen alteraciones en la función inmunológica que tienen su origen debido principalmente a influencias genéticas, ya que son los genes de respuesta inmunitaria funcional los responsables de la susceptibilidad a asma múltiple y enfermedad atópica. Como base científica para el estudio de información se puede considerar al consorcio GABRIEL que realizó un estudio de asociación genómica del asma, este identificó una asociación significativa para IL18R1, IL33, HLA-DQ, SMAD3, IL2RB y ORMDL3. 
Encontraron que a pesar de que cada uno de estos genes tiene una gran variedad de funciones, todos se encontraban involucrados en la respuesta inflamatoria Th2 al daño epitelial sostenido durante un trauma o infección. También se ha postulado que el asma - la alergia de los padres puede influir en la función inmunológica, con antecedentes maternos de atopia o asma identificados como un factor de riesgo para enfermedades más graves asociadas con VPH. Pero, quizás la evidencia más sólida de una relación entre el aumento de la susceptibilidad a la infección entre las personas con asma y la enfermedad atópica es el aumento de las tasas de enfermedad invasiva, demostrando un mayor riesgo de enfermedad neumocócica grave en personas con enfermedad atópica. Las personas con dermatitis atópica también son altamente susceptibles a infecciones víricas diseminadas, como el eccema mollusculatum, el eccema herpético y el eccema vaccinatum [45].

Tal fue el caso de estudio que examinaba la asociación entre el asma y la enfermedad neumocócica invasiva. El grupo de estudio involucró a personas de 2 a 49 años que se inscribieron en el programa de Medicaid de Tennesse. Se identificaron un total de 635 personas con enfermedad neumocócica invasiva y 6350 controles, de los cuales $114(18 \%)$ y $516(8,1 \%)$ respectivamente, tenían asma. Las personas con asma tenían un mayor riesgo de enfermedad neumocócica invasiva en comparación con los controles [46].

La presencia del asma debido a condiciones geográficas ha sido motivo de diversos estudios, sin embargo, en recientes estudios se ha visto una relación existente entre el aumento de la gravedad del asma y una concentración elevada de especies fúngicas alergénicas, una alta concentración total de hongos y una alta riqueza bacteriana, además de la composición de la comunidad microbiana. La gravedad del asma en niños atópicos se asoció con la composición de la comunidad de hongos, mientras que la gravedad del asma en niños no atópicos se asoció con la concentración total de hongos [47].

\subsection{Obesidad}

Para hablar sobre la obesidad y su relación con la diversidad de microorganismos, el microbioma o el microbioma es necesario considerar el primer estudio que desarrollo pistas claves sobre el papel del microbioma en la patogénesis de la obesidad. En el estudio desarrollado por Backhed et al. compararon el aumento de peso corporal en ratones libres de gérmenes y ratones criados convencionalmente, y encontraron que estos últimos aumentaron de peso, con un aumento del porcentaje de grasa en el tejido adiposo y corporal, lo que no podría explicarse por la ingesta de diferentes dietas. En sí, el estudio y lo demás estudios que lo presidieron en modelos animales establecieron que no todos los microbiomas tienen el mismo efecto sobre el metabolismo y plantearon la posibilidad de una microbiota específica para la obesidad. Entonces, a partir de esto es que varios grupos intentaron caracterizar la microbiota asociada a la obesidad, sin embargo, estudios realizados en la obesidad humana dieron resultados diferentes a los estudios en animales agregando una menor diversidad microbiana y diferencias similares en la microbiota intestinal [47]. 
Es un hecho la relación existente entre la microbiota intestinal y la obesidad, y es justamente por esto que esta última se puede transmitir de modo infeccioso mediante el trasplante del microbioma, lo que sugiere que es la microbiota la que promueve la obesidad. Y este concepto se logró evaluar mediante el estudio prospectivo en niños, este mostró que el riesgo de sobrepeso a los siete años podría predecirse por la composición de la microbiota intestinal a los seis meses de edad, asociando ésta a una menor prevalencia de Bifidobacterium y una mayor de Staphylococcus aureus [48].

Debido a la alta tasa de obesidad y sus complicaciones derivadas como la diabetes, se ha prestado especial atención a la comprensión del mecanismo subyacente. Durante la última década, han surgido nuevos estudios que sugieren un papel para la microbiota intestinal en el desarrollo de la obesidad y la diabetes. Los primeros estudios mostraron que los ratones obesos y los humanos tenían una composición microbiana diferente en comparación con la magra. Otro ejemplo del papel de la microbiota en la obesidad se ha visto en pacientes sometidos a bypass gástrico, en los cuales después de la cirugía se manifiesta una mejoría metabólica dramática que no puede explicarse por la restricción calórica y la pérdida de peso solo. Se ha demostrado que los cambios en el microbioma intestinal desempeñan un papel en esta mejora, ya que en varios estudios se ha observado un cambio en la población bacteriana [49].

También se demostró que el microbioma intestinal desempeña un papel en la regulación de los ácidos biliares y el metabolismo del colesterol en humanos y animales. Los ácidos biliares se sintetizan en el hígado por una vía multietapa. Es posible que la intervención del microbioma intestinal en el control del metabolismo de los ácidos biliares y del colesterol podría ser inducido por la regulación al alza de los factores de transcripción que lo vinculan con la inflamación inducida por la nutrición, la absorción de lípidos y la lipogénesis de Novo. De ser posible, se debe tener en cuenta el uso de modalidades de tratamiento dirigidas a los cambios de comportamiento en el caso de la obesidad hedónica y aquellas que pueden modificar el punto de ajuste del peso corporal en el caso de la obesidad metabólica. Finalmente, aunque no se pueda cambiar la genética subyacente a la obesidad metabólica o hedónica, se puede cambiar el entorno obesogénico que favorece el desarrollo de estas afecciones [50].

\subsection{Trastornos del neurodesarrollo}

Existen muchos estudios que relacionan el microbioma intestinal que conecta al intestino con la red cerebral, y se ha tratado de conectar esta relación con problemas de carácter neurológico altamente complejos como el autismo y la esquizofrenia. Sin embargo, traducir estos prometedores beneficios preclínicos a trastornos del desarrollo neurológico humano es un desafío, y no hay pruebas suficientes de una relevancia clínica sólida [50].

En la esquizofrenia, hay una evidencia preliminar emergente de un microbioma intestinal alterada. Pero aún se requieren extensos estudios clínicos intervencionistas prospectivos más grandes, con marcadores centrales de la función cerebral, que utilicen la modulación terapéutica del microbioma intestinal o sus metabolitos [51]. 
La esquizofrenia es otro trastorno complejo del comportamiento heterogéneo caracterizado por un comportamiento social anormal, a menudo asociado con problemas adicionales de salud mental, como trastornos de ansiedad y depresión mayor. Existe evidencia de la relación entre diversos factores ambientales y el desarrollo de la esquizofrenia. Hay indicaciones, como el hecho de que la dopamina, el neurotransmisor clave asociado con la fisiopatología y el tratamiento de la esquizofrenia, es producida por microbios, y la inflamación gastrointestinal asociada con la esquizofrenia, lo que sugiere fuertemente que el microbioma intestinal está involucrado en el riesgo de esquizofrenia o desarrollo sus manifestaciones [52].

La identificación de los mecanismos epigenéticos y transgeneracionales en los trastornos del desarrollo neurológico mediados por infección parece relevante para los trastornos cerebrales independientemente de las clasificaciones diagnósticas existentes y puede ayudar a identificar patrones complejos de transmisión de enfermedades transgeneracionales más allá de la herencia genética. La consideración de historias infecciosas ancestrales puede ser de gran interés clínico y puede ser fundamental para el desarrollo de nuevas estrategias de tratamiento preventivo contra los trastornos del desarrollo neurológico mediados por infección [53].

\section{Conclusiones}

Las bacterias, son microorganismos que han logrado colonizar la gran mayoría de las superficies terrestres, mostrando una gran capacidad de adaptación.

Estando el ser humano constituido por estructuras orgánicas cuyas superficies también han sido colonizadas por estos microorganismos, desde la misma vida intrauterina, manteniendo una relación de aparente simbiosis, delimitando la proliferación de aquellos organismos patógenos. De igual forma, se ha planteado múltiples hipótesis aseverando la gran importancia del equilibrio del microbioma humano en el desarrollo de la salud e inicio de la enfermedad.

\section{Agradecimiento}

Un especial agradecimiento a la Escuela Superior Politécnica de Chimborazo, y a la Escuela de Medicina de la Facultad de Salud Pública por las herramientas brindadas para nuestra formación académica. Y al apoyo recibido por parte de nuestros familiares, quienes han sido los pilares fundamentales en nuestro camino.

\section{Conflictos de Interés}

El grupo de autores declaran, no tener conflicto alguno de interés. 


\section{Limitaciones de Responsabilidad}

Los autores declaramos que todos los puntos de vista expresados en el presente documento son de nuestra entera responsabilidad y no de la institución en la que laboramos.

\section{Fuentes de Apoyo}

La financiación del presente documento proviene de los mismos autores.

\section{References}

[1] Cho I, Blaser MJ. The human microbiome: At the interface of health and disease. Nature Reviews Genetics. 2012;13:260-70.

[2] Turnbaugh PJ, Hamady M, Yatsunenko T, et al. A core gut microbiome in obese and lean twins. Nature. 2009;457(7228):480-4.

[3] Wang J, Qin J, Li Y, et al. A metagenome-wide association study of gut microbiota in type 2 diabetes. Nature. 2012;490(7418):55-60.

[4] Gensollen T, Blumberg RS. Correlation between early-life regulation of the immune system by microbiota and allergy development. Journal of Allergy and Clinical Immunology. 2017;139:1084-91.

[5] Turnbaugh PJ, Ley RE, Hamady M, Fraser-Liggett CM, Knight R, Gordon Jl. The Human Microbiome Project. Nature. 2007;449: 804-10.

[6] Peterson J, Garges S, Giovanni M, et al. The NIH Human Microbiome Project. Genome Res. 2009;19(12):2317-23.

[7] Qin J, Li R, Raes J, Arumugam M, et al. A human gut microbial gene catalogue established by metagenomic sequencing. Nature. 2010;464(7285):59-65.

[8] 'Ome sweet' omics- a genealogical treasury of words. National Library of Medicine. Available from: https://lhncbc.nlm.nih.gov/publication//hncbc-2001-047

[9] Mackie RI, Sghir A, Gaskins HR. Developmental microbial ecology of the neonatal gastrointestinal tract. Am J Clin Nutr. 1999;69(5):1035s-1045s.

[10] Dominguez-Bello MG, Blaser MJ, Ley RE, Knight R. Development of the human gastrointestinal microbiota and insights from high-throughput sequencing. Gastroenterology. 2011;140(6):1713-9.

[11] Dominguez-Bello MG, Costello EK, Contreras M, et al. Delivery mode shapes the acquisition and structure of the initial microbiota across multiple body habitats in newborns. Proc Natl Acad Sci U S A. 2010;107(26):11971-5.

[12] Palmer C, Bik EM, DiGiulio DB, Relman DA, Brown PO. Development of the human infant intestinal microbiota. PLoS Biol. 2007;5(7):e177.

[13] Koenig JE, Spor A, Scalfone N, et al. Succession of microbial consortia in the developing infant gut microbiome. Proc Natl Acad Sci U S A. 2011;108(SUPPL. 1):4578-85.

[14] Fierer N, Lauber CL, Zhou N, McDonald D, Costello EK, Knight R. Forensic identification using skin bacterial communities. Proc Natl Acad Sci U S A. 2010;107(14):6477-81.

[15] Ursell LK, Metcalf JL, Parfrey LW, Knight R. Defining the human microbiome. Nutr Rev. 2012;70(Suppl. 1):S38.

[16] DiGiulio DB, Romero R, Amogan HP, et al. Microbial prevalence, diversity and abundance in amniotic fluid during preterm labor: A molecular and culture-based investigation. PLoS One. $2008 ; 3(8)$.

[17] DiGiulio DB. Diversity of microbes in amniotic fluid. Seminars in Fetal and Neonatal Medicine. 2012;17:211.

[18] Goldenberg RL, Culhane JF. Infection as a cause of preterm birth. Clinics in Perinatology. 2003;30:677_ 700.

[19] van Gorp C, de Lange IH, Spiller OB, et al. Protection of the ovine fetal gut against ureaplasma-induced chorioamnionitis: A potential role for plant sterols. Nutrients. 2019;11(5)

[20] Kwak DW, Hwang HS, Kwon JY, Park YW, Kim YH. Co-infection with vaginal Ureaplasma urealyticum and Mycoplasma hominis increases adverse pregnancy outcomes in patients with preterm labor or preterm premature rupture of membranes. J Matern Neonatal Med. 2014;27(4):333-7. 
[21] Menon R, Dunlop AL, Kramer MR, Fortunato SJ, Hogue CJ. An overview of racial disparities in preterm birth rates: Caused by infection or inflammatory response? Acta Obstetricia et Gynecologica Scandinavica. 2011;90: 1325-31.

[22] Steel JH, Malatos S, Kennea N, et al. Bacteria and inflammatory cells in fetal membranes do not always cause preterm labor. Pediatr Res. 2005;57(3):404-11.

[23] Satokari R, Grönroos T, Laitinen K, Salminen S, Isolauri E. Bifidobacterium and Lactobacillus DNA in the human placenta. Lett Appl Microbiol. 2009;48(1):8-12.

[24] Aagaard K, Ma J, Antony KM, Ganu R, Petrosino J, Versalovic J. The placenta harbors a unique microbiome. Sci Transl Med. 2014;6(237):237-65.

[25] Nuriel-Ohayon M, Neuman H, Koren O. Microbial changes during pregnancy, birth, and infancy. Frontiers in Microbiology. 2016;7:1031.

[26] Biedermann L, Rogler G. The intestinal microbiota: Its role in health and disease. European Journal of Pediatrics. 2015;174:151-67.

[27] Gosalbes MJ, Llop S, Vallès Y, Moya A, Ballester F, Francino MP. Meconium microbiota types dominated by lactic acid or enteric bacteria are differentially associated with maternal eczema and respiratory problems in infants. Clin Exp Allergy. 2013;43(2):198-211.

[28] Hu J, Nomura Y, Bashir A, et al. Diversified microbiota of meconium is affected by maternal diabetes status. PLoS One. 2013;8(11).

[29] Arrieta MC, Stiemsma LT, Amenyogbe N, Brown E, Finlay B. The intestinal microbiome in early life: Health and disease. Frontiers in Immunology. 2014;5.

[30] Jiménez E, Marín ML, Martín R, et al. Is meconium from healthy newborns actually sterile? Res Microbiol. 2008;159(3):187-93.

[31] Ardissone AN, de la Cruz DM, Davis-Richardson AG, et al. Meconium microbiome analysis identifies bacteria correlated with premature birth. PLoS One. 2014;9(3):e90784.

[32] Gosalbes MJ, Llop S, Vallès Y, Moya A, Ballester F, Francino MP. Meconium microbiota types dominated by lactic acid or enteric bacteria are differentially associated with maternal eczema and respiratory problems in infants. Clin Exp Allergy. 2013;43(2):198-211.

[33] Bokulich NA, Chung J, Battaglia T, et al. Antibiotics, birth mode, and diet shape microbiome maturation during early life. Sci Transl Med. 2016;8(343):343-82.

[34] Navarro CW. La lactancia materna y sus propiedades microbioinmunológicas. Rev del Cuerpo Médico Hosp Nac Almanzor Aguinaga Asenjo. 2011;4(1):63-6.

[35] Rodríguez JM, Jiménez E, Merino V, Maldonado A, et al. Microbiota de la leche humana en condiciones fisiológicas. Acta Pediatr Esp. 2008;66(2):77-82.

[36] Ariza R, García M. El microbioma humano. Su papel en la salud y en algunas enfermedades. Cir Cir. 2012;80(4):128-33.

[37] Deyanira D, Rosa Hernández L, José E, Cabeza G, Niurka D, Castañeda S. La microbiota intestinal en el desarrollo del sistema inmune del recién nacido Intestinal microbiota in the development of the neonate's immune system. Revista Cubana de Pediatría. 2014;86.

[38] Prince AL, Antony KM, Chu DM, Aagaard KM. The microbiome, parturition, and timing of birth: More questions than answers. J Reprod Immunol. 2014;104-105(C):12-9.

[39] Brunser O. El desarrollo de la microbiota intestinal humana, el concepto de probiótico y su relación con la salud humana. Rev Chil Nutr. 2013;40.

[40] Serrano CA, Harris PR. Development of intestinal microbiome in children. Impact on health and disease. Rev Chil Pediatr. 2016;87(3):151-3.

[41] Zamudio-Vázquez VP, Ramírez-Mayans JA, Toro-Monjaraz EM, et al. Importancia de la microbiota gastrointestinal en pediatría. Acta Pediatr Mex. 2017;38(1):49-62.

[42] Torres J, Espinosa LL, García ÁM, Mideros AM, Usubillaga E. Características de recién nacidos con enterocolitis necrotizante en un hospital universitario de tercer nivel en Colombia. Colomb Med. 2011;42(4):468-75.

[43] Mena V, Riverón R, Pérez J, Paz B. Factores de riesgo asociados a la mortalidad por enterocolitis necrotizante. Rev Cuba Pediatr. 1998;70.

[44] Pacuruco AL, Carchi CM, Jaramillo KS, Beckman CV. Parámetros de laboratorio, indicadores de gravedad en prematuros con enterocolitis necrotizante. Rev Med Ecuador. 2009;15(3):213-21.

[45] James KM, Peebles RS, Hartert T V. Response to infections in patients with asthma and atopic disease: An epiphenomenon or reflection of host susceptibility? J Allergy Clin Immunol. 2012;130(2):343-51.

[46] Talbot TR, Hartert T V, Mitchel E, et al. Asthma as a risk factor for invasive pneumococcal disease. N Engl J Med. 2005;352(20):2082-90.

[47] Pekkanen J, Valkonen M, Täubel M, et al. Indoor bacteria and asthma in adults: A multicentre casecontrol study within ECRHS II. Eur Respir J. 2018;51(2). 
[48] Machado MV, Cortez-Pinto H. Diet, microbiota, obesity, and NAFLD: A dangerous quartet. International Journal of Molecular Sciences. 2016;17:1-20.

[49] Baothman OA, Zamzami MA, Taher I, Abubaker J, Abu-Farha M. The role of Gut Microbiota in the development of obesity and diabetes. Lipids in Health and Disease. 2016;15

[50] Yu YH, Vasselli JR, Zhang Y, Mechanick JI, Korner J, Peterli R. Metabolic vs. hedonic obesity: A conceptual distinction and its clinical implications. Obesity Reviews. 2015;16:234-47.

[51] Cenit MC, Sanz Y, Codoñer-Franch P. Influence of gut microbiota on neuropsychiatric disorders. World Journal of Gastroenterology. 2017'23:5486-98.

[52] Kelly JR, Minuto C, Cryan JF, Clarke G, Dinan TG. Cross talk: The microbiota and neurodevelopmental disorders. Frontiers in Neuroscience. 2017;11.

[53] Weber-Stadlbauer U. Epigenetic and transgenerational mechanisms in infection-mediated neurodevelopmental disorders. Translational Psychiatry. 2017;7: 1113. 